\title{
Uncovering How Viruses Evade Cell Defenses
}

\author{
Biophysicist James Hurley studies how viruses, such as the one that \\ causes COVID-19, manipulate cellular membranes to infect cells.
}

\author{
By Erika K. Carlson
}

$\int$ ames Hurley is known to his peers as a structural biologist. But it was in physics, not biology, that he got his start in science. After earning his bachelor's degree in physics, Hurley entered a biophysics Ph.D. program to study molecular structures. Now, he researches interactions between cellular membranes and proteins-including those created by viruses-as a structural biologist at the University of California, Berkeley. He cites many factors for inspiring his decision to pursue biology and biophysics, including a desire to work on topics more closely tied to society and everyday life, such as AIDS-the 1980s AIDS epidemic took hold in the US when Hurley was beginning his research career. Hurley spoke with Physics about his work and about what physicists can bring to biology research.

All interviews are edited for brevity and clarity.

\section{What is the focus of your research?}

I'm particularly fascinated by cellular structures that need to

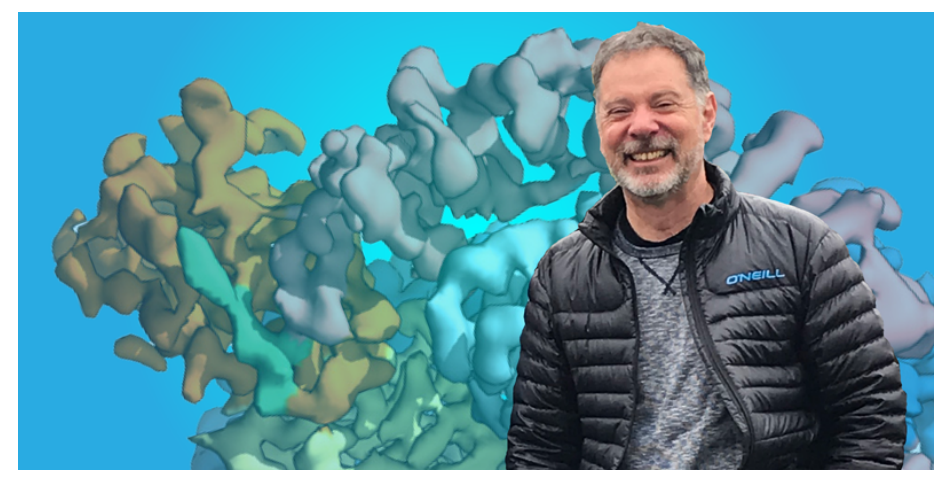

Credit: J. Hurley; adapted by APS/Alan Stonebraker change shape rapidly over time. The most far-out example of this phenomenon is probably autophagy, which is when a cell swallows up and destroys some waste product or foreign matter. During this process, a double-membrane vesicle-which starts out looking like a cup and then grows into a roughly spherical shape-forms de novo in the cell in a matter of minutes. And this vesicle can engulf and eat other things in the cell, including invading pathogens.

Interactions between cell membranes and pathogens become even more interesting when you consider that intercellular pathogens also manipulate cell membranes-for example, to cross the membrane. Their mechanisms can be elaborate and can eliminate a cell's defenses rather than just slip through them.

\section{What have you learned about pathogens and cellular defenses?}

In apes and monkeys, there is an endemic ancestor of the HIV virus called SIV. It's been around for hundreds of thousands of years, but it only passed to humans as HIV about 100 years ago. My collaborators and I determined the molecular structures that explain how humans had been able to avoid the virus, and therefore AIDS, for so long.

Humans share more than $99 \%$ of our DNA with chimpanzees, but there are a few significant differences. One of them is in a protein called tetherin, which affects our susceptibility to viruses. Tetherin is a defense molecule that restricts the ability of viruses to replicate. In apes and monkeys, SIV targets a specific piece of this defense molecule, holding onto it like a handle and then destroying the molecule. But in humans, that 
piece of tetherin is missing; we have somehow removed the handle that SIV uses to undermine ape and monkey defenses.

My group didn't discover the "handle" mechanism. But we came up with a picture of the handle that really highlights the molecular differences in ape and monkey versus human tetherin.

\section{You are also working on the virus that causes COVID-19. Can you share what your group is investigating?}

Coronaviruses in general, not just SARS-CoV-2, which causes COVID-19, replicate in membrane-delimited compartments within host cells. So we're very interested in how coronavirus proteins work to reshape host-cell membranes. And, like HIV, coronaviruses have ways of undermining host defenses-SARS-CoV-2, for example, also grabs onto handles in our defense proteins and disarms them.

There are thousands of other coronaviruses in bats and other animals that have not crossed over to humans. Others have induced only mild colds, and more serious ones, such as SARS and MERS, were quickly contained. What's the difference between them and the pandemic-causing SARS-CoV-2? One thing, we think, is that SARS-CoV-2 has developed quicker, more potent ways to undermine our host defenses. So we are looking at that to see if this coronavirus has any unique invasion mechanisms that are absent in others.

Finding such a mechanism won't necessarily lead to a treatment, but I think it would be helpful for understanding how this disease came to be so bad.

\section{Where does physics show up in your work?}

It's everywhere. It's in the forces that dictate biomolecular interactions. It's in the probes that we use to determine molecular structures-primarily, electron microscopy and x-ray diffraction. And it's in the shapes of membranes. For example, at low resolution, we use elasticity theory to calculate how much energy it takes to bend the membrane into a particular shape. To study these phenomena at higher resolution, we use molecular simulations, which are also based on these forces of physics.

\section{Do you have any advice for physics students interested in a career in biophysics or biology research?}

An undergraduate degree in physics is a fantastic springboard into a biology or biophysics Ph.D. It often helps if the student has completed a couple of biological courses or volunteered in a biologically oriented lab, but that isn't essential. Transitioning at the postdoc level is also possible. One of my star postdocs had a Ph.D. in physics and had never touched any sort of biological equipment before joining my lab.

There are many opportunities for people who have strong computational and math skills and who are interested in understanding how life is organized or in curing diseases. Jump on over, the water's warm.

Erika K. Carlson is a Corresponding Editor for Physics based in New York City. 